\title{
Corela
}

Cognition, représentation, langage

HS-32 | 2020

Les postures énonciatives

\section{Dénomination et postures énonciatives autour d'islam dans la presse quotidienne française : le poids des attentats}

Dénomination et postures énonciatives autour d'islam dans la presse quotidienne française : le poids des attentats

\section{Alice Pitoizet et Francis Grossmann et Agnès Tutin}

\section{(2) OpenEdition}

\section{Journals}

Édition électronique

URL : https://journals.openedition.org/corela/12312

DOI : $10.4000 /$ corela. 12312

ISSN : 1638-573X

\section{Éditeur}

Cercle linguistique du Centre et de l'Ouest - CerLICO

Référence électronique

Alice Pitoizet et Francis Grossmann et Agnès Tutin, « Dénomination et postures énonciatives autour d'islam dans la presse quotidienne française : le poids des attentats », Corela [En ligne], HS-32 | 2020, mis en ligne le 05 novembre 2020, consulté le 14 juillet 2021. URL : http://journals.openedition.org/ corela/12312; DOI : https://doi.org/10.4000/corela.12312

Ce document a été généré automatiquement le 14 juillet 2021.

\section{(†)}

Corela - cognition, représentation, langage est mis à disposition selon les termes de la licence Creative Commons Attribution - Pas d'Utilisation Commerciale - Partage dans les Mêmes Conditions 4.0 International. 


\section{Dénomination et postures énonciatives autour d'islam dans la presse quotidienne française : le poids des attentats}

Dénomination et postures énonciatives autour d'islam dans la presse quotidienne française : le poids des attentats

Alice Pitoizet et Francis Grossmann et Agnès Tutin

\section{Introduction}

1 Durant la période récente, les médias se sont trouvés confrontés à des problèmes de dénomination délicats, liés à l'utilisation du terme islam et à ses sous-catégorisants. À côté d'oppositions sémantiques traditionnelles, telles qu'islam vs islamisme (Seniguer, 2012), on trouve fréquemment aujourd'hui des séquences nom-adjectif (islam radical, islam modéré, islam religieux, islam politique) ou $\mathrm{N}$ de $\mathrm{N}$ (islam de France, islam des caves) qui entrent dans un microsystème terminologique en voie de constitution. Cependant, ces dénominations sont loin d'être stabilisées dans un sens précis, et leur contexte d'énonciation, tout comme l'usage qu'en font les locuteurs, est déterminant pour leur interprétation. Elles traduisent une "catégorisation identitaire par contraste" (Steuckardt, 2002: 7) qui peut se complexifier, lorsque «l'autre » auquel on se réfère est présenté lui aussi comme clivé, contrasté. Nous nous situerons dans une perspective argumentative et dialogique, afin de mieux comprendre, comment se sont traduits, au sein du discours de presse, les tensions et les débats liés au rôle de l'islam. L'analyse, issue de l'étude d'un corpus de presse couvrant les années 2014 et 2015, avant et après les attentats de Charlie Hebdo et du Bataclan, se répartit en deux volets :

- un décompte des occurrences des syntagmes comportant, pour la période étudiée, le pivot islam, dans l'ensemble du corpus ${ }^{1}$, suivi d'une analyse des syntagmes les plus productifs, prenant en compte le statut de l'énonciateur (politiques, journalistes ou organisations). On s'intéresse en particulier à l'une des 
dénominations les mieux représentées : islam de France et à son usage en contexte. L'on s'efforce de préciser, à l'aide d'une analyse du contexte d'emploi, à quelles fins argumentatives elles sont mobilisées ;

- une étude qui se penche plus spécifiquement sur le cas des éditoriaux figurant dans le corpus et analyse le jeu des postures énonciatives entre les différents énonciateurs représentés; l'éditorialiste, qu'il exprime un point de vue sur un événement pris en charge collectivement par la rédaction (Le Monde), individuellement par le directeur de la rédaction (Libération) ou encore par l'une des signatures du journal (Le Figaro) peut sembler mettre, de manière privilégiée, le locuteur/énonciateur primaire en posture de sur-énonciation; nous chercherons à savoir si cette hypothèse se vérifie, et comment cette voix primaire dialogue avec les énonciateurs qu'elle cite ou représente.

2 L'objectif de la recherche est de voir comment le point de vue ${ }^{2}$ impliqué par la sélection lexicale (islam et ses qualifications) peut être éclairé en contexte par a) l'analyse du cotexte (approche classique en lexicométrie), puis complété par b) l'analyse des postures énonciatives dans les énoncés qui mobilisent les dénominations ou caractérisations d'islam.

\section{Méthodologie}

3 Le corpus étudié regroupe tous les articles des journaux Le Monde, Le Figaro et Libération contenant la forme islam ou le lemme musulman (incluant le pluriel ou le féminin du nom et de l'adjectif) entre le $1^{\mathrm{er}}$ janvier 2014 et le 31 décembre $2015^{3}$. Tous les termes morphologiquement liés à islam n'ont pas été retenus. Nous avons exclu islamisme, par exemple, qui, en raison de sa polarité négative, est déjà argumentativement marqué. Le corpus a été réparti en trois sous-corpus : le premier, intitulé " pré Charlie Hebdo » (01/01/2014-07/01/2015) couvre la période avant l'attentat qui a eu lieu au siège de ce journal, le deuxième "post Charlie Hebdo » (08/01/2015-13/11/2015) et le troisième, intitulé " post Bataclan » (14/11/2015-31/12/2015), après l'attentat qui s'est déroulé dans la salle de spectacle parisienne. Le sous-corpus 1 (pré Charlie), recueilli sur une durée d'un an, comporte 1803213 mots. Le sous-corpus 2 (post Charlie) est composé de 15934737 mots, avec tous les articles recueillis durant une période de 10 mois. Le souscorpus 3 (post Bataclan), le moins important, contient 349005 mots et a été recueilli sur 1 mois et demi. Pour l'étude effectuée ici, nous avons réuni les trois sous-corpus.

Enfin, l'analyse des postures énonciatives concerne 49 éditoriaux issus de ce même corpus, clairement identifiés en tant que tels, comportant en tout 68 mentions d'islam (pour environ 29460 mots). Nous nous appuierons en particulier sur les notions de point de vue (Rabatel, 2003, 2015a), de posture énonciative (Rabatel, 2012 et 2015b), et plus précisément les postures de sur-énonciation, sous-énonciation et co-énonciation (Rabatel, 2004, 2007, 2012 2015b).

5 En croisant deux méthodes d'analyse, nous chercherons à montrer, à partir de l'étude du corpus, comment l'approche pragmatico-énonciative liée aux postures peut venir compléter et enrichir une analyse de type lexico-sémantique fondée sur l'observation des dénominations d'islam et sur leur fonctionnement en contexte. 


\section{Le « paysage » lexical des SN comportant islam}

\subsection{Inventaire des syntagmes intégrant islam comme pivot}

6 Dans une démarche d'analyse lexicologique nous avons observé les contextes immédiats des occurrences d'islam afin d'extraire les collocations les plus productives incluant ce mot en tant que pivot. Nous avons utilisé la recherche de cooccurrences de l'outil en ligne Anatext ${ }^{4}$ avec la requête islam $+\mathrm{ADJ}$ et la requête islam de $\mathrm{N}$ afin d'observer les syntagmes les plus employés. Le tableau 1 indique les cooccurrences les plus productives pour ces associations :

\begin{tabular}{|c|c|c|c|}
\hline Islam Adj & Fréquence & Islam de $\mathrm{N}$ & Fréquence \\
\hline islam radical & 509 & islam de France & 304 \\
\hline islam politique & 141 & islam des Lumières & 22 \\
\hline islam sunnite & 103 & islam des origines & 19 \\
\hline islam modéré & 53 & islam de tolérance & 8 \\
\hline islam chiite & 32 & islam du juste milieu & 8 \\
\hline islam rigoriste & 26 & islam de paix & 8 \\
\hline islam français & 22 & islam des caves & 6 \\
\hline islam contemporain & 16 & islam d'Europe & 5 \\
\hline islam traditionnel & 16 & & \\
\hline islam fondamentaliste & 15 & & \\
\hline islam tolérant & 11 & & \\
\hline islam républicain & 10 & & \\
\hline islam authentique & 10 & & \\
\hline islam dévoyé & 10 & & \\
\hline islam wahhabite & 10 & & \\
\hline
\end{tabular}

TABLEAU 1 : Les syntagmes nominaux les plus fréquents (au moins 5 occ.) comportant islam

7 Certaines des structures islam + ADJ, (islam radical, islam politique, islam sunnite...) peuvent être considérées comme des dénominations, dans la mesure où elles sont utilisées avec des référents relativement stables. Elles s'inscrivent souvent dans les séries s'organisant dans des oppositions (chiite vs sunnite, modéré vs radical, ...). L'emploi habituel de l'article défini singulier accompagnant ces expressions (l'islam radical, l'islam modéré) confirme leur statut de dénomination en renvoyant de façon non ambiguë à 
une réalité partagée. Dans les termes de type $N+A D J$, certains adjectifs à l'origine qualifiants (modéré, radical) ont perdu leur statut caractérisant pour construire des classes conceptuelles. Toutefois, plusieurs de ces dénominations restent ambiguës et ne peuvent être interprétées qu'en contexte. L'expression islam français par exemple désigne simplement l'islam pratiqué en France dans l'expression un des meilleurs connaisseurs de l'islam français (Le Figaro, entretien avec Michel Godard, 19/02/2015) alors que dans l'exemple (1), elle désigne l'islam en cours d'institutionnalisation sur le territoire français et a un statut de dénomination :

(1) Pour l'élu UMP, l'Islam français doit «s'organiser de manière plus représentative », le conseil français du culte musulman « évoluer »... (Le Monde, 15 février 2015).

Certaines dénominations renvoient à des notions assez spécifiques, qui ne sont pas immédiatement accessibles. Le terme islam des caves, par exemple, réfère aux pratiques de l'islam hors de lieux de cultes institutionnels, faute de mosquées. Les dénominations islam des Lumières ou islam des origines, si elles renvoient à des périodes spécifiques de l'Islam, ont aussi des emplois référentiels assez flous ${ }^{5}$.

9 L'ensemble des dénominations peut être réparti en plusieurs grands groupes (cf. Fig.1). On peut tout d'abord mentionner les dénominations « internes ", celles qui permettent aux musulmans (et aux historiens en général) de spécifier les différentes familles ou branches de l'islam. Il s'agit de l'opposition chiite-sunnite et des différents mouvements religieux, principalement orthodoxes (islam salafiste, islam wahhabite...). Un deuxième grand groupe représente les dénominations externes qui correspondent à une évaluation de la part de l'énonciateur primaire. Parmi celles-ci apparaissent des expressions à connotation négative, qui semblent majoritaires en nombre et en fréquence (islam radical, islam intégriste, islam fondamentaliste...) et des dénominations plus rares à connotations positives (islam tolérant, islam modéré). Le dernier groupe intègre les dénominations de nature plus institutionnelle, qu'il s'agisse des notions employées par des historiens (islam des Lumières), les politiques ou les organismes religieux (islam de France, islam républicain) ou les sociologues (islam identitaire).

Image

10051C9C000081DD0000385AA2F87FC3BC6533D5.emf

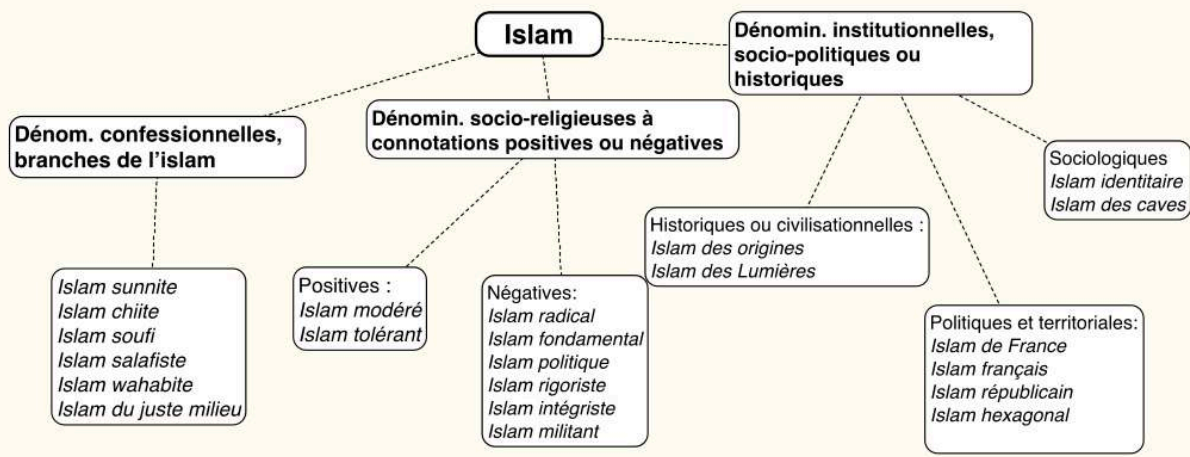

FIg. 1 : Typologie des dénominations les plus fréquentes avec islam

10 À côté de ces dénominations, qui renvoient à des concepts stables ou en cours de stabilisation, on observe des expressions moins lexicalisées qui renvoient à des caractérisations de l'islam ou à des désignations plus ponctuelles. Les principaux qualifiants présents relèvent du champ lexical de la pureté (islam pur) souvent associé 
au salafisme, distinct de l'islam authentique, véritable que chaque courant semble revendiquer. Les qualifiants à connotation positive sont ici plus nombreux que dans le cas des dénominations (islam de paix, islam de tolérance, islam éclairé, islam moderne, islam ouvert), souvent associés au déterminant un. Quelques qualifiants à polarité négative apparaissent toutefois (islam conquérant, islam dévoyé) à côté d'adjectifs moins évaluatifs renvoyant à des âges plus anciens de l'islam (islam classique, islam traditionnel) ou récents (islam contemporain). Étant donné le nombre important d'expressions spécifiques, il n'est pas envisageable de proposer une étude approfondie des contextes et des connotations associés à chacune de ces expressions. Nous avons donc choisi de nous intéresser plus en détail aux emplois d'une dénomination emblématique, islam de France.

\subsection{Le cas d'islam de France}

Le syntagme islam de France est le plus productif parmi les co-occurrents compléments du nom islam de type « de $+\mathrm{N}$ », et peut être considéré comme dénomination au sens de Kleiber (1984 et 2001). Il est difficile de retrouver, s'il a existé vraiment, l'«acte de baptême » de l'expression. En tout cas, elle n'est pas référencée dans les dictionnaires Larousse, Robert ou sur le TLF. Sur l'encyclopédie collective en ligne Wikipédia, on trouve uniquement l'entrée islam en France. De plus, le syntagme qui nous intéresse est largement utilisé dans la presse et donc par extension, dans le discours politique (quand les journalistes rapportent la parole de politiciens), mais il est très peu usité par les chercheurs. Il ne fait en tout cas l'objet d'aucun consensus définitoire stable. Plusieurs articles de presse ou blog s'interrogent sur le sens que véhicule cette expression. Courrier international la présente comme renvoyant à un « islam propre à la France ». Nasser Suleiman, dans son blog sur le Huffington Post ${ }^{7}$, questionne la formule : «L'islam de France existe-t-il ? Cette dénomination prête à discussion. [...] est-elle liée à une juste appréciation des choses? Ou est-elle guidée par la peur?». Il propose néanmoins une clarification : islam de France renverrait à « une religion insérée ayant pour charge de garantir l'institution dans une parfaite adéquation avec l'espace des cultes en France » tandis qu'islam en France serait «caractérisé par un islam, en mal d'organisation, déterminé par les logiques d'états et de pouvoirs étrangers ». Nicolas Sarkozy a lui aussi joué sur l'opposition des deux dénominations lors du débat de l'entre-deux-tours le 3 mai $2012^{8}$, en affirmant vouloir « un islam de France et non un islam en France ». L'ancien président de la République a même revendiqué l'invention du terme islam de France lors d'un déplacement le 8 avril 2016. Mais, comme le précise Alain Auffray dans l'éditorial de Libération du même jour", "l'expression est dans le débat public depuis plus d'un quart de siècle. La plupart des ministres de l'intérieur qui ont précédé Sarkozy l'ont utilisée, à commencer par le socialiste Pierre Joxe, créateur en 1990 d'un "Conseil de réflexion sur l'Islam de France" ». La notion d'islam de France a également fait l'objet d'un rapport du Sénat publié en $2016^{10}$. Ce dernier n'interroge pas concrètement l'usage de la formule et traite avant tout des questions d'organisation institutionnelle de l'islam en France. Le texte précise quand même dans sa conclusion qu'il a pour objectif de "suggérer des pistes de transition vers un Islam de France, adapté au contexte français, à la fois compatible avec les valeurs de la République française et pris en charge par la communauté elle-même: renouvellement de la représentation, formation en France des imams, formation laïque obligatoire des imams détachés et des aumôniers, transparence accrue du financement, notamment 
étranger ». En bref, pour bon nombre de locuteurs de la sphère médiatique et politique, la dénomination islam de France reste une "formule rassurante totalement floue " (Belkaïd et Schmid, 2015). Elle n'est fondée sur aucune doctrine politique, pas plus qu'elle ne recoupe un corpus théologique précis. En utilisant les contextes des occurrences islam de France, nous tenterons nous aussi de préciser les acceptions de cette dénomination en essayant autant que faire se peut de différencier les énonciateurs. Nous avons regroupé les cooccurrents de la collocation selon leurs isotopies sémantiques au sein du corpus.

\subsubsection{Diversité ou division}

12 Les syntagmes relevés en Fig. 2 mettent en évidence une première isotopie, celle de la diversité, voire de la division. Un ensemble de substantifs appartenant au réseau sémantique de l'hétérogénéité est utilisé, comme tendances dans les syntagmes 4 et 5 , courants en 2 , branches en 3 et composantes en 1 . D'après ces exemples, l'identité référentielle de la collocation islam de France est composée de plusieurs sous-catégories et est donc présentée comme plurielle. Pourtant, on remarque que le syntagme nominal qui nous intéresse est systématiquement déterminé ici par un article défini. Le déterminant défini est ici utilisé dans son emploi générique et implique donc des connaissances partagées par le locuteur et l'allocutaire (le lecteur) comme si le renvoi référentiel à d'islam de France était connu de tous. D'un point de vue énonciatif, les syntagmes incluant à la fois islam de France et un substantif lié au champ de la division sont principalement pris en charge directement par les journalistes. On les retrouve moins dans les discours indirects rapportant la parole de politiciens, associatifs ou intellectuels. En outre, d'après l'ensemble des exemples cités, le syntagme islam de France est utilisé par les journalistes sans modalisation, ce qui montre qu'il semble intégré dans le discours de presse.

\begin{tabular}{|l|l|l|}
\hline Division & Syntagme relevé & Type d'énonciateur \\
\hline 1 & les principales composantes de l'islam de France & Journaliste \\
\hline 2 & les principaux courants de l'islam de France & Journaliste \\
\hline 3 & l'une des branches de l'islam de France & Journaliste \\
\hline 4 & toutes les tendances de l'islam de France & NSP \\
\hline 5 & Des organisations issues de différentes tendances de l'islam de France & Journaliste \\
\hline 6 & l'islam de France, qui est divers et éclaté & Intellectuel \\
\hline
\end{tabular}

Fig. 2. L'isotopie de la diversité et de la division dans les co-occurrents d'islam de France

\subsubsection{Rassemblement et union}

Dans quelques exemples du corpus prévaut l'isotopie sémantique de l'union à travers l'emploi des verbes fédérer, rallier et se rassembler. Deux de ces verbes sont employés dans des constructions infinitives précédées de pour en 9 et 10 et islam de France est 
sujet de se rassembler dans une construction avec l'auxiliaire pouvoir, ici synonyme de être capable de, dans l'exemple 11. Ces observations ajoutent l'idée d'objectif à celle d'union. Bien sûr, même si l'énonciateur est ici journaliste, cela ne veut pas dire que c'est lui qui souhaite directement l'union de l'islam de France.

\begin{tabular}{|l|l|l|}
\hline Union & Syntagme relevé & Type d'énonciateur \\
\hline 9 & CFCM, créé pour fédérer l'islam de France & Journaliste \\
\hline 10 & pour rallier l'islam de France à & Journaliste \\
\hline 11 & montrer que l'islam de France pouvait se rassembler & Journaliste \\
\hline
\end{tabular}

Fig. 3. L'isotopie du rassemblement et de l'union

\subsection{3. Émergence et construction}

Plus fréquemment encore que l'union, c'est l'isotopie de l'émergence et de la construction (Fig.4) qui est mobilisée quand le discours de presse traite de la question de l'islam de France. Le syntagme est en effet complément d'objet de construire en 12 et en 19, de devenir en 17 et de faire émerger en 15. Il est également complément du nom de construction en 20. Ces exemples s'opposent à ceux précédemment cités (cf. division et union) car ils présupposent paradoxalement que l'islam de France n'existe pas et que son référent doit être construit. Enfin certains exemples $(13,14,18$ et 21$)$ complexifient encore davantage le sens que revêt islam de France avec cette fois des cooccurrents appartenant au réseau sémantique du changement, avec l'emploi de réforme et réformer en 13 et 21 et réorganisation en 18. Les lignes définitionnelles d'islam de France sont donc particulièrement floues témoignant de la polysémie du terme : le syntagme renvoie à la fois à un référent présenté comme hétérogène et dont on souhaite l'unicité, à une entité qu'il faut construire ou encore à un ensemble que l'on veut réformer. On peut donc analyser tous ces emplois d'islam de France comme renvoyant à des questionnements métalinguistiques mais aussi sociaux et politiques.

Enfin, il est intéressant de noter que les politiciens sont les principaux utilisateurs des mots construction, faire émerger ou encore réformer. Cette prise en charge énonciative peut révéler une volonté avant tout politique de restructurer l'islam pratiqué en France.

\begin{tabular}{|l|l|l|}
\hline $\begin{array}{l}\text { En } \\
\text { construction }\end{array}$ & Syntagme relevé & Type d'énonciateur \\
\hline 12 & Dans l'espoir de construire un islam de France & Politique \\
\hline 13 & dans le cadre de la réforme de l'islam de France & Journaliste \\
\hline 14 & Un islam de France... appelé à se réformer & Politique (Valls) \\
\hline 15 & notre incapacité à faire émerger un islam de France & Politique (Larcher) \\
\hline
\end{tabular}




\begin{tabular}{|l|l|l|}
\hline 16 & $\begin{array}{l}\text { Il faut arriver à construire quelque chose de solide, pour } \\
\text { qu'il y ait enfin un vrai islam de France }\end{array}$ & Religieux (imam) \\
\hline 17 & ce que l'islam peut faire pour devenir l'islam de France & Politique (Sarkozy) \\
\hline 18 & $\begin{array}{l}\text { Rien ne se fera sans une réorganisation complète de } \\
\text { l'islam de France }\end{array}$ & Associatif \\
\hline 20 & $\begin{array}{l}\text { il s'agit donc de construire un véritable islam de France } \\
\text { on a trop tardé dans la construction de l'islam de France } \\
\text { l'armée }\end{array}$ & $\begin{array}{l}\text { Politique (Karim El El } \\
\text { Karoui) }\end{array}$ \\
\hline 21 & $\begin{array}{l}\text { le gouvernement avait réaffirmé l'importance de de } \\
\text { «réformer l'islam de France » }\end{array}$ & journaliste \\
\hline
\end{tabular}

Fig. 4. L'isotopie de l'émergence et de la construction

\subsubsection{L'assise institutionnelle}

À l'encontre de ce qui a été observé précédemment, certaines propositions relevées dans le corpus inscrivent l'islam de France dans l'isotopie de la stabilité, associée à l'assise institutionnelle.

17 Selon le discours des journalistes et d'associatifs, l'islam de France a des représentants en 23 et 26 , des autorités officielles en 22 et des figures respectées en 28. L'aspect institutionnel (mis en lumière par la fig. 5) est présent avec le mot instance $(25,27)$ au singulier ou au pluriel.

\begin{tabular}{|l|l|l|}
\hline Stabilité institutionnelle & Syntagme relevé & Type d'énonciateur \\
\hline 22 & autorités officielles de l'islam de France & journaliste \\
\hline 23 & les représentants de l'islam de France & journaliste \\
\hline 24 & les responsables de l'islam de France & $\begin{array}{l}\text { imam/associatif } \\
\text { journaliste }\end{array}$ \\
\hline 25 & le CFCM, l'instance qui représente l'islam de France & journaliste \\
\hline 26 & les représentants des musulmans de France & journaliste \\
\hline 27 & les instances représentatives de l'islam de France & associatif \\
\hline 28 & Figures respectées de l'islam de France & journaliste \\
\hline
\end{tabular}

Fıg. 5. L'isotopie de l'assise institutionnelle 


\subsubsection{L'isotopie volontariste et injonctive}

Comme nous l'avons vu en 2.2.4., les définitions d'islam de France sont différentes selon que les énonciateurs se situent au plan du constat ou d'une analyse politique projective : en l'état actuel, l'islam de France est divisé, mais il doit s'unir à l'avenir ; il est seulement émergent, mais doit déjà être réformé ; bien qu'il ait des représentants institutionnels, il doit parachever sa structuration. Le discours prescriptif et normatif (Fig.6) qui lui est adressé s'explique essentiellement par cette demande d'institutionnalisation. Cela explique aussi que l'islam soit vu davantage comme une structure que comme une communauté de croyants ou de pratiquants.

Plusieurs verbes déontiques sont utilisés parmi les co-occurrents d'islam de France ou des expressions qui réfèrent. En 31, le syntagme n'est pas présent en tant que tel, mais l'assertion selon laquelle l'islam « est en France pour y rester » se trouve dans la phrase qui précède immédiatement celle qui contient l'injonction. Le verbe devoir en 36 et 37 a comme sujet le syntagme étudié. Ce dernier est aussi sujet de la proposition subordonnée complétive complément du verbe impersonnel falloir (35).

On note également l'emploi de verbes de requête vouloir en 32, pousser en 33 et exhorter en 34, islam de France étant en position d'objet. Les sujets de ces trois verbes sont respectivement nous renvoyant à Nicolas Sarkozy qui est ici l'énonciateur, le Premier ministre et le gouvernement. Tous ces syntagmes renvoient à la politique. On remarque d'ailleurs que les énonciateurs qui utilisent ces verbes d'injonction ou de requête sont souvent des politiciens ${ }^{11}$.

\begin{tabular}{|l|l|l|}
\hline $\begin{array}{l}\text { Injonction/ } \\
\text { requête }\end{array}$ & Syntagme relevé & $\begin{array}{l}\text { Type } \\
\text { d'énonciateur }\end{array}$ \\
\hline 29 & $\begin{array}{l}\text { Cette irruption de l'islam le plus violent oblige les composantes } \\
\text { de l'islam de France à prévenir toute radicalisation }\end{array}$ & journaliste \\
\hline 30 & Ceux qui doivent représenter l'IDF & journaliste \\
\hline 31 & $\begin{array}{l}\text { L'islam est en France pour y rester. Il faut mener le combat des } \\
\text { consciences }\end{array}$ & Politique (Valls) \\
\hline 32 & $\begin{array}{l}\text { Nous voulons un islam de France et pas un islam en France } \\
\text { (Sarkozy) }\end{array}$ \\
\hline 34 & Le gouvernement pousse l'islam de France à se réorganiser & journaliste \\
\hline 36 & $\begin{array}{l}\text { le premier ministre a exhorté « l'islam de France à se prendre } \\
\text { en main » }\end{array}$ & Politique (Valls) \\
\hline Il faut que l'islam de France assume & politique \\
\hline
\end{tabular}




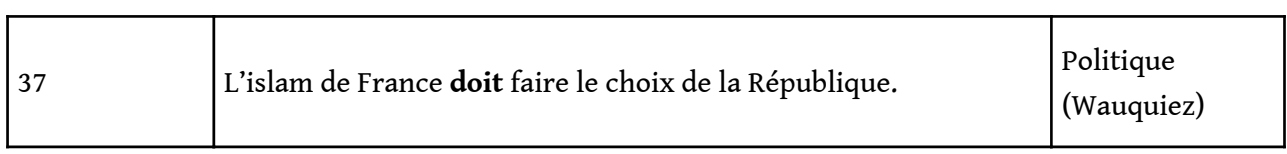

Fig. 6. L'isotopie volontariste et injonctive

L'entité référentielle d'islam de France, dans les réseaux lexicaux analysés, est présentée comme soumise à des forces qui le contraignent. La notion ne renvoie pas aux membres d'une communauté (les fidèles de l'islam en France), mais plutôt à un objet institutionnel, envisagé en dehors des pratiques religieuses (aucun terme de ce champ sémantique n'est présent), dont se saisit la sphère politique. Enfin le rapport à l'islam, tel qu'il se marque lexicalement et à travers les structures prédicatives, est fondamentalement ambivalent : l'islam est vu comme divisé, mais appelé à s'unifier, comme n'existant pas réellement, puisqu'il faut construire le "véritable » islam, et l'édifier au plan institutionnel. L'éthos volontariste qui va de pair avec les injonctions et requêtes qu'exprime ou relaie le discours de presse, semble avoir des affinités avec une posture énonciative de sur-énonciation. C'est ce que nous allons vérifier à travers le cas particulier du discours des éditorialistes.

\section{Des dénominations aux postures énonciatives : les éditoriaux}

\subsection{Rappel de quelques caractéristiques de l'éditorial}

L'éditorial est un genre mouvant et hétérogène (Fløttum, 1998), dans lequel les discours rapportés abondent (Fløttum, 2001). Dans sa thèse, M. Roitman a étudié dans une perspective énonciative un corpus d'éditoriaux issus des mêmes journaux que les nôtres (Le Figaro, Le Monde, Libération), mais sur une période antérieure. Elle rappelle (2006: 1) quelques caractéristiques des éditoriaux : ils ne rapportent pas simplement un événement, mais présentent des opinions sur un événement particulier, souvent déjà connu des lecteurs. Cela explique leur polyphonie constitutive et leur caractère fréquemment intertextuel, puisque, s'appuyant sur la mémoire discursive du lecteur, ils citent ou font allusion à d'autres discours circulant dans l'espace social, concernant l'événement relaté. Fløttum (1998 et 2001) avait déjà montré que l'hétérogénéité énonciative peut être considérée comme un trait constitutif des éditoriaux. Les critères utilisés par Roitman (2006 : 18-19), à partir de manuels de journalisme, d'encyclopédies de presse et d'interviews avec des journalistes pour définir dans sa thèse l'éditorial, sont les suivants :

1) il s'agit d'un texte relativement court;

2) il occupe un espace privilégié dans le journal ;

3) il porte souvent le titre « éditorial »;

4) il traite un sujet à la fois ;

5) il a un message précis ;

6) c'est un sujet de portée large sur des problèmes de société ou sur la vie politique ;

7) sa rédaction est (souvent) anonyme et collective ;

8) le destinataire est collectif et très diversifié ;

9) il a une disposition argumentative ;

10) sa visée est la persuasion ;

11) il exprime la position sociopolitique du journal. 
Nous ne retiendrons pas le critère 7 , dans la mesure où nous avons également inclus dans le corpus « éditoriaux » des textes qui expriment le point de vue, mis en vedette (cf. critère 2) d'un éditorialiste régulier du journal, comme c'est le cas pour Le Figaro. Dans ce cas, on peut considérer que l'éditorialiste qui signe représente, si ce n'est le point de vue de la rédaction, au moins une tendance qui s'exprime en son sein. Comme le rappelle Ringoot (2015: 215), «l'identité collective du journal n'exclut pas pour autant la personnalisation par des signatures et des réputations individualisées » : c'est ce qui explique que certains journaux aient plusieurs éditorialistes, aux opinions parfois différentes.

\subsection{La répartition des différents syntagmes dénominatifs}

La figure 7 ci-dessous permet de visualiser les fréquences des différents syntagmes dénominatifs comprenant le mot islam ${ }^{12}$ :

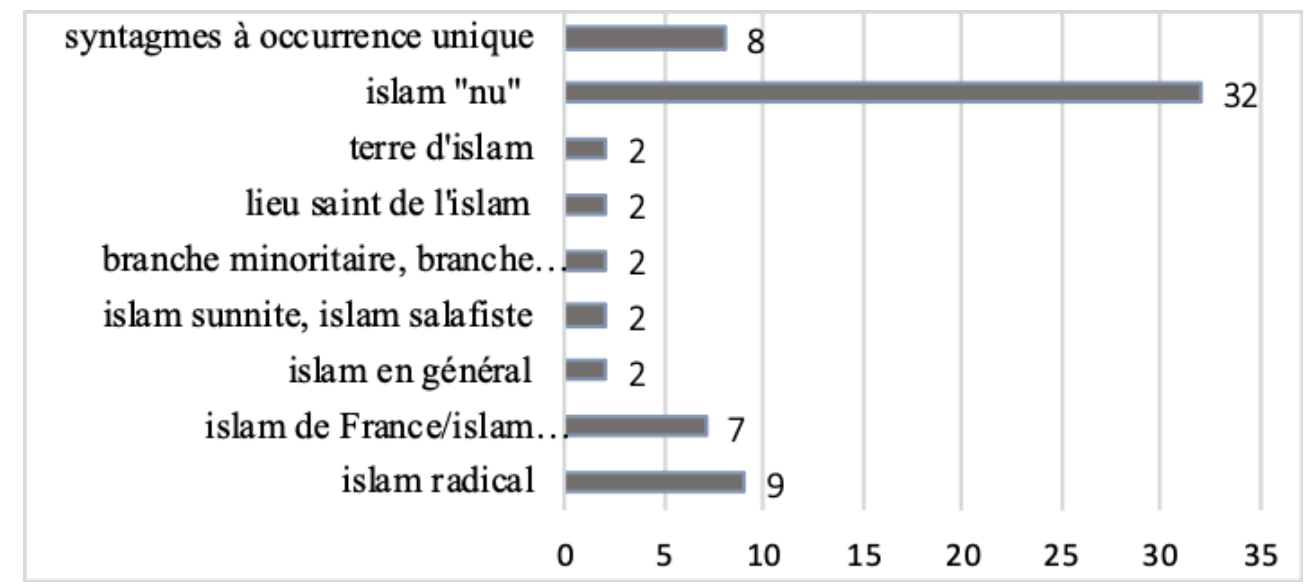

Fıg. 7 : Les SN comportant le mot islam dans les éditoriaux $(n=66)$

Quelques points intéressants peuvent être notés. Tout d'abord le fait, peu surprenant en soi, que le vocable islam est majoritairement employé «nu » (sans modification par un adjectif ou un complément déterminatif). Nous reviendrons ci-après sur les caractéristiques de cet emploi dans les éditoriaux.

En ce qui concerne cette fois les syntagmes, on peut noter une certaine dispersion, puisque 8 occurrences, listées ci-dessous, ne se retrouvent qu'une seule fois dans les éditoriaux : islam républicain, l'Armée de l'Islam, l'islam d'aujourd'hui, islam fondamentaliste, islam politique, islam des Lumières, le vrai islam, islam modéré.

Cette dispersion se voit également dans le fait que 6 autres types de catégorisation ne comportent que 2 occurrences (malgré des regroupements, ex. branche minoritaire / majoritaire de l'islam). Par rapport aux différents types de dénominations mentionnés dans la partie générale (cf. Fig. 1), on note que les dénominations « internes ", celles qui permettent aux musulmans (et aux historiens en général) de spécifier les différentes familles ou branches de l'islam sont peu nombreuses. Dans les éditoriaux, s'il est parfois question du monde sunnite, ou du monde chiite, ou encore des sunnites et des chiites, on n'a trouvé qu'une mention d'islam sunnite, et aucune d'islam chiite, ce qui s'explique en partie par le fait que l'islam est très majoritairement sunnite en France. Bien que les références au salafisme soient assez nombreuses (le discours des salafistes, le danger salafiste...), la dénomination islam salafiste n'a été trouvée qu'une seule fois également, 
ce qui peut laisser penser que la référence au salafisme est davantage utilisée, de manière externe, comme une catégorie repoussoir, que comme une dénomination précise désignant un mouvement religieux. L'islam dont il est question dans les éditoriaux reste dans l'ensemble peu spécifié, au plan religieux comme au plan géopolitique ${ }^{13}$. Ce qui intéresse le plus les éditorialistes - et cela peut se comprendre dans le contexte des attentats - c'est plutôt l'ancrage dans la situation française, et les menaces que ferait peser un islam « radical ». C'est d'ailleurs le syntagme islam radical qui est le plus fréquent (9 occurrences). Son antonyme islam modéré ne se retrouve qu'une seule fois. Ce sont les dénominations islam de France, islam en France, islam français qui arrivent ensuite avec 7 occurrences.

Ce sont donc les catégorisations " externes ", celles qui impliquent une évaluation de la part de l'énonciateur primaire, qui sont très majoritaires. Face à islam radical, nettement péjoratif, on a vu que ce qui est présenté comme positif dans les éditoriaux, à travers des dénominations comme islam de France, islam en France, c'est moins la modération (une seule occurrence d'islam modéré) que l'enracinement dans la vie publique française et donc la capacité pour l'islam d'être compatible avec les pratiques sociales et culturelles majoritaires. Un éditorial du Monde, en date du 25 février 2015, thématise plus directement cette question: "Cela fait vingt-cinq ans que la question est posée : la République laïque doit-elle - et comment - aider les Français musulmans à s'organiser pour consolider l'expression d'un « islam de France »? ». Les guillemets de modalisation montrent le caractère émergent à la fois de la dénomination et du référent qui lui correspond, selon les journalistes. La dénomination islam des Lumières peut être considérée à la fois comme interne et comme externe : elle est interne dans la mesure où elle émane de penseurs et théologiens réformistes issus de l'islam, mais elle est souvent utilisée par les journalistes occidentaux pour montrer qu'un pont est possible entre la république et l'islam ${ }^{14}$.

\subsection{Dénominations et argumentation}

Les dénominations, et les sous-catégorisations qu'elles impliquent, nourrissent l'argumentation. Les éditorialistes recourent aux formes classiques du raisonnement (ex. 2, en forme de syllogisme), mais aussi à des postulats implicites, fondés sur des représentations sociales ou des stéréotypes (ex. 3) :

(2) La plupart des musulmans ne sont pas salafistes. Mais les salafistes sont une branche extrême, fondamentaliste, de l'Islam. (Libération, 23/11/2015)

(3) Si près de 2000 Français «seulement " - si l'on ose dire - sont militairement formés au djihad, ils sont dix fois plus ${ }^{15}$ à être intellectuellement formés par l'islam salafiste. (Le Figaro, 28/06/2015)

En (2), il s'agit de montrer que le salafisme, même s'il est minoritaire, appartient bien à l'islam. En (3), l'argumentation implicite, à travers la structure concessivo-adversative introduite par si (marqueur d'altérité énonciative selon Monte, 2009) et par rapprochement des deux chiffres fournis, est qu'il existe un lien entre les deux catégories, l'islam militaire des djihadistes et le salafisme. En suggérant que le salafisme conduit au djihadisme, l'éditorialiste minore le point de vue adverse, qui voudrait minimiser le poids du djihadisme en France. La sur-énonciation se marque à travers la principale, qui réoriente le point de vue, mais aussi, dans la subordonnée, à travers la mise entre guillemets de seulement et le commentaire méta-énonciatif si l'on ose dire. Au bout du compte, l'inférence produite est que les 2000 Français djihadistes ne 
représentent que la partie émergée de l'iceberg et que la fabrique du terrorisme islamiste peut compter sur un bien plus grand nombre.

\subsection{Les postures énonciatives des éditorialistes}

31 L'éditorial est fait pour présenter un point de vue surplombant sur un fait d'actualité et a donc des affinités évidentes avec la sur-énonciation. L'étude des postures énonciatives dans les éditoriaux confirme, sans surprise, la prédominance de la posture de sur-énonciation de l'énonciateur primaire (désormais L1/E1). Cette posture de surplomb dans les contextes comportant islam se marque en particulier à travers les catégorisations généralisantes qui fonctionnent par contraste, et qu'assume ou rejette l'énonciateur primaire, en marquant son accord ou son désaccord avec les énonciateurs secondaires cités ou représentés. À partir des éditoriaux analysés, nous distinguons deux niveaux d'analyse : d'une part, la posture de sur-énonciation vs sous-énonciation (qui marque la position de surplomb ou au contraire d'allégeance de L1/E1 par rapport à un autre énonciateur); d'autre part, l'évaluation du point de vue prêté à autrui : accord, désaccord (ou bien non marquage) avec parfois leurs corrélats pathémiques (empathie, rejet, colère...). Nous nous proposons d'illustrer les procédés utilisés à travers quelques exemples significatifs.

\subsubsection{Sur-énonciation et discours rapporté}

Les éditorialistes adoptent fréquemment une posture de sur-énonciation, par rapport aux discours qu'ils rapportent. Dans les exemples (4) et (5) ci-dessous, le syntagme l'islam en général ${ }^{16}$ est mobilisé dans le discours lorsqu'il est question du risque d'amalgamer l'islam-religion avec l'islamisme, sa déviation « radicale » ou sectaire. Le même syntagme est mobilisé en discours avec une orientation argumentative inverse, à travers le même topos du refus de l'amalgame.

(4) La mise en cause de l'islam en général, au nom d'une supposée "guerre des civilisations ", la critique obsessionnelle du "politiquement correct " qui refuse d'assimiler tous les musulmans à des ennemis est le troisième axe de l'offensive qui vise à défendre «l'identité malheureuse » du pays en dénonçant, de front ou de biais, la minorité musulmane. (Libération, 20/11/2015)

(5) Car le caractère implacable du constat dressé par Valls révèle en creux le caractère incantatoire de la mise en garde de Hollande contre les «vaines querelles ». En appelant un chat un chat, c'est-à-dire en nommant le danger salafiste et en prévenant de la nature durable de sa menace, le Premier ministre fait apparaitre la part de déni dans un discours qui, par crainte de l'amalgame avec l'islam en général, s'interdit d'employer les mots adéquats. (Le Figaro, 20/06/2015)

Dans l'éditorial de Libération, le discours de surplomb vise un discours prêté, de manière assez allusive, à de nombreux politiques et des intellectuels omniprésents; le caractère critique du discours tenu par ces énonciateurs se note à travers les marques de distance ou évaluatives (supposée "guerre de civilisation", critique obsessionnelle, et l'usage des guillemets de modalisation). Dans l'éditorial du Figaro, le jeu énonciatif est plus complexe puisque L1/E1 vise à la fois à saluer les positions exprimées par Manuel Valls, alors Premier ministre (E2), tout en stigmatisant le discours tenu habituellement par le président de la République - et au-delà par la gauche - (E3) qui s'interdit d'employer les mots adéquats. L1/E1 marque son accord avec le discours tenu par E2 et son désaccord par rapport à celui de E3. 


\subsubsection{Sur-énonciation et polémique : la dévalorisation du point de vue d'autrui} par l'adverbe bref: la dénomination islam radical est intégrée dans un énoncé dont l'arrière-plan assertif (selon les mécanismes de la présupposition) est que l'islam radical a une présence grandissante en France. Une autre forme d'ironie se marque dans la reprise feinte dans un éditorial du Monde des réactions que peut avoir un énonciateur "naif " (ici à la deuxième personne, de manière à impliquer davantage le lecteur) devant la polémique sur la place accordée à l'islam dans les programmes scolaires $^{17}$ :

(8) Mercredi 29 avril, ce sont les nouveaux programmes d'histoire du collège qui ont suscité une autre polémique. Selon certains, ils accorderaient trop de place à l'enseignement du fait musulman au Moyen Âge, au détriment du christianisme. Vous n'en savez rien, vous croyiez que l'histoire du monde musulman occupait, déjà, une place logique et légitime dans les programmes. Mais vous en retenez que, à l'évidence, l'islam dérange, même dans les livres d'histoire. (Le Monde, 8/05/2015)

La co-énonciation entre le point de vue de l'éditorialiste et le "naïf » mis en scène (qui n'en sait rien) débouche sur le constat «évident " que l'islam dérange, même dans les livres d'histoire : on retrouve alors in fine le style assertif de la sur-énonciation, qui permet de dominer le point de vue prêté aux dénonciateurs des nouveaux programmes. 


\subsubsection{La sous-énonciation et son rôle dans l'argumentation} énonciation, qui n'apparaissent généralement dans les éditoriaux que comme une stratégie provisoire, comme le montrent l'ex. 9, ainsi que l'ex. 14, analysé plus loin.

(9) Bien sûr, les spécialistes ont cent fois raison d'exhorter l'opinion à la précision, à ne pas confondre salafistes et Frères musulmans, piétistes et jihadistes, à rappeler inlassablement que l'immense majorité des Français de culture musulmane sont étrangers à l'islam radical. Distinctions élémentaires à l'heure où de nombreux politiques et des intellectuels omniprésents s'acharnent à jeter la suspicion sur la minorité musulmane. Mais cette maladie de l'islam qu'est l'intégrisme pose trois questions : [...] (Libération, 23/11/2015).

La position apparente de sous-énonciation de L1/E1 qui s'abrite derrière le discours des experts (les spécialistes ont cent fois raison de...) introduit en fait un mouvement concessif précédant le signalement des «questions» que pose cette maladie de l'islam (cf. la présupposition déjà analysée induite par le complément déterminatif) et dont l'énoncé reprend quant à lui le style assertif de la sur-énonciation.

Une autre forme de sous-énonciation, plus subtile, se fonde sur la fusion du point de vue de L1/E1 avec celui de l'énonciateur qu'il met en scène, ce qui correspond à la coénonciation dans la terminologie de Rabatel (2007 : 90 ${ }^{18}$. Ainsi, dans l'exemple (10), issu du Figaro, le point de vue de l'éditorialiste est difficile à démêler de celui de l'énonciateur secondaire. L'éditorialiste rapporte la trame du roman Soumission de Houellebecq (nous soulignons les passages qui permettent de mieux saisir les formes que revêt ici le dialogisme) :

(10) Houellebecq n'élève jamais le ton pour décrire ce qui semble inéluctable : la transformation de la France en un pays musulman. L'action se situe en 2022. À la faveur d'un duel avec Marine Le Pen, soutenu par l'alliance des partis traditionnels, un fin stratège prêchant un islam modéré accède à l'Élysée. Il contrôle l'enseignement. Spécialiste d'Huysmans, le narrateur, après quelques vaines tentatives de ressourcement spirituel, se soumet à ce nouveau régime. Les convertis y sont bien traités et ont droit à la polygamie. C'est tout de même plus confortable que d'avoir à courir les prostituées. Houellebecq surfe sur les peurs contemporaines, et en premier lieu celle de l'islam. On le lui reproche. Accusé de souffler sur les braises, il rétorque que son livre est un roman, une politique-fiction, librement inspirée du réel. De quoi serait-il coupable ? Il livre sa vision de l'avenir. Elle a des allures de cauchemar. Il n'en fait pas un drame. Son héros paraît même s'en accommoder. Il n'éprouve aucune sympathie pour personne; plutôt de l'apathie. Son épuisement est le miroir de nos démissions. (Le Figaro, 06/01/2015)

Dans la première phrase, L1/E1 résume la trame du livre, et sa thèse implicite. La position de l'énonciateur principal est ambiguë : on ne sait pas s'il accepte ou non la thèse prêtée à Houellebecq, qui décrit «ce qui semble inéluctable ${ }^{19}$ ». Deux phrases semblent orienter l'éditorial dans deux directions opposées : l'éditorialiste signale que Houellebecq surfe sur les peurs contemporaines (remarque qui peut sembler critique) et qu'on le lui reproche. Ce on rassemble les détracteurs du roman qui y ont vu, à tort ou à raison, un brûlot contre l'islam. Sont présentées ensuite, sans commentaire, les justifications du romancier (il s'agit de politique fiction et non de réalité). Mais la phrase, qui fournit une interprétation à l'apathie du héros, Son épuisement est le miroir de nos démissions semble bien, quant à elle, assumée par L1/E1. Cette phrase, qui a valeur conclusive $^{20}$, fournit une clé d'interprétation à l'ensemble des propos rapportés ${ }^{21}$ permettant ainsi à l'éditorialiste de reprendre la main et de se placer en surplomb. 


\subsubsection{Retour sur islam de France}

avons vu en 3.2. les ambiguïtés attachées à l'expression lorsqu'elle est mobilisée dans le discours de presse. Comment est-elle employée dans les éditoriaux ? Nous nous proposons d'analyser plus finement les postures énonciatives dans les contextes dans lesquels elle apparaît ${ }^{22}$.

\section{Libération : une posture claire de sur-énonciation}

L'éditorial, en date du 23 novembre 2015, se situe après l'attentat du Bataclan (qui a eu lieu le 13 novembre). Il a été rédigé par Laurent Joffrin, et est intitulé Amalgame.

(11) [...] Qui mieux que les musulmans peuvent combattre la maladie de leur propre religion? Il faut encourager les musulmans qui ont décidé, avec courage, de s'élever contre l'islam radical. Les autorités représentatives se sont engagées dans cette voie. Il appartient à l'État français de les conforter en assurant à cet islam des conditions décentes d'exercice du culte. Cette bataille est décisive pour l'avenir de l'islam de France - et pour la République. Plus que par des mises en garde extérieures, c'est en voyant des musulmans s'engager que l'opinion évitera l'amalgame qui fait tant de mal. (Libération, 23/11/2015)

L'argumentation repose sur le présupposé, marqué lexicalement à travers la construction déterminative (combattre la maladie de leur propre religion) selon lequel l'islam radical est une maladie de l'islam. La conséquence de ce raisonnement c'est que, d'après l'éditorialiste, c'est aux musulmans eux-mêmes de régler le problème de l'islamisme radical. Ce point de vue se précise à travers les cinq rôles énonciatifs mis en scène par l'énonciateur primaire, au sein d'un discours narrativisé : a) les musulmans qui ont décidé de s'élever avec courage contre l'islam radical et qui «doivent être encouragés "; b) les « autorités représentatives » qui se sont engagées dans cette voie ; c) l'État français qui doit «les conforter»; d) les tenants de "mises en garde extérieures », jugées inefficaces ; e) l'« opinion » qui pourra éviter les amalgames entre islam et islam radical si elle voit les musulmans s'engager contre l'islam radical.

L1/E1 a clairement une posture de sur-énonciation, qui se manifeste à travers les déontiques analysés en 3.2. (Il faut..., Il appartient à) ; l'éditorialiste distribue les bons points avec mesure (les autorités représentatives (= de l'islam de France) se sont engagées dans cette voie) (cf. l'isotopie de la construction, analysée en 3.2.). L'injonction adressée par l'éditorialiste est donc double : elle s'adresse d'abord aux musulmans, qui doivent lutter contre l'islamisme en s'engageant clairement contre lui - par le biais d'institutions représentatives; elle s'adresse ensuite à l'État français qui doit fournir une assise institutionnelle (l'islam de France). Le modèle rêvé (un islam de France intégré à la République) voudrait le faire reposer sur un modèle proche d'autres religions, par exemple celui de l'Église réformée de France. L'opinion, voix anonyme de la majorité silencieuse des Français, est présentée comme susceptible de (continuer à) faire l'amalgame «qui fait tant de mal» entre les deux islams en cas de non réponse à l'injonction. L'éditorialiste mobilise cette voix anonyme de l'opinion pour donner plus de poids à sa propre injonction.

\section{Le Monde : entre sous et co-énonciation}

L'éditorial du Monde date quant à lui du 25 février 2015, soit plus d'un mois après les attentats de janvier. L'expression islam de France apparaît deux fois, au début et à la fin 
de l'éditorial, et est explicitement thématisée (dans les deux cas) à partir du mot question, qui introduit également un discours rapporté narrativisé - dont la source énonciative n'est pas identifiable. Cette question est explicitée dès la première phrase :

(12) Cela fait vingt-cinq ans que la question est posée : la République laïque doit-elle - et comment - aider les Français musulmans à s'organiser pour consolider l'expression d'un « islam de France » ? Un islam fidèle aux valeurs de la République. Capable de répondre aux multiples problèmes cultuels et culturels qui se posent à la seconde religion du pays. Déterminé, enfin, à opposer un message de tolérance à ceux qui veulent le dévoyer et invoquent Allah pour justifier un fanatisme dévastateur. Mais cela fait vingt-cinq ans que les gouvernements, de gauche comme de droite, ne parviennent pas à trouver une réponse satisfaisante. (...) (Le Monde, $25 / 02 / 2015)$

Les guillemets qui encadrent islam de France peuvent marquer le fait que l'expression islam de France circule dans l'espace social, mais qu'elle n'est pas stabilisée. La suite permet d'en définir un contenu (fidèle aux valeurs de la République. Capable de répondre (...). Déterminé enfin...) dont L1/E1 (l'éditorialiste) semble prendre en charge la définition, en syncrétisme avec ceux qui cherchent à " consolider l'expression » de cet islam de France. La posture énonciative est donc très différente de celle adoptée par Libération dans l'éditorial précédent. Elle oscille entre sous-énonciation (le passif à valeur impersonnelle ne permet pas de savoir qui pose ou a posé la question) et coénonciation. La suite confirme cette posture empathique, l'éditorialiste donne son aval (la démarche (...) est donc salutaire) par le ministre de l'Intérieur de l'époque (B. Cazeneuve), dont les propos sont repris et largement cités :

(13) La démarche annoncée par le président de la République et le Premier ministre au lendemain des attentats parisiens de janvier, et désormais engagée par le ministre de l'Intérieur, est donc salutaire. Bernard Cazeneuve entend, en effet, bâtir autour du CFCM « une instance la plus représentative possible » regroupant imams de terrain, intellectuels, universitaires et associations. Chargée d'offrir un lieu de dialogue aux musulmans de France dans leur diversité, elle devra également répondre à des problèmes concrets, laissés en jachère depuis des années: la formation universitaire des imams, ainsi que celle des aumôniers musulmans intervenant dans les prisons, la place des écoles confessionnelles, la question de l'abattage rituel (halal) ou celle des fêtes religieuses. Les écueils ne manquent pas, à commencer par le risque d'enfermer les musulmans dans une " communauté » qui les isolerait plus qu'elle ne favoriserait leur intégration dans la société française. Mais, selon la formule de M. Cazeneuve, des "compromis intelligents" sont possibles, nécessaires, urgents même. Sauf à laisser la question de l'islam de France s'envenimer, pour le plus grand bénéfice de tous les démagogues qui veulent en faire un sujet de crispation, de tension et de rejet. (Le Monde, 25/02/2015)

La dernière phrase, qui semble à nouveau plus directement prise en charge par l'éditorialiste anonyme du Monde, vient compléter la «formule» de M. Cazeneuve en lui donnant une portée plus dramatique, mais qui reste en convergence avec la teneur du discours de ce dernier ; la question de l'islam de France risque en effet de s'envenimer si on ne trouve pas les compromis intelligents proposés par ce dernier. Certes, l'éditorialiste a le dernier mot, mais il choisit de mettre en évidence la co-énonciation avec celui qui, selon lui, évalue au plus juste les modalités et les résultats de l'action publique qu'il convient d'entreprendre. 


\section{Le Figaro : une feinte posture de co et sous-énonciation} attentats de janvier. On retrouve le discours injonctif, associé cette fois à une forte dimension normative ( il faudra bien un jour fixer les règles») qui, en creux, dénonce l'absence de règles actuelles :

(14) Autre chantier, l'islam de France, que Nicolas Sarkozy avait voulu organiser sans y parvenir. Il faudra bien un jour fixer des règles pour éviter que le premier imam autoproclamé, par des prêches enfiévrés, suscite et encourage des vocations de djihadistes. Dans les mosquées comme sur Internet. Bref, ces journées sanglantes nous obligent à tout revoir, à tout repenser. Il s'agit, entre autres choses, du sempiternel débat entre la liberté ou la sécurité. Depuis la semaine dernière, on croit sentir de quel côté penchent les Français. (Le Figaro, 12/01/2015)

Ce discours normatif contredit l'apparente posture de sous-énonciation, que semblent induire le on et l'auxiliaire à valeur modale (on croit sentir), voire de co-énonciation (l'éditorialiste implique le lecteur grâce au nous). Ces marqueurs entrent en fait dans une rhétorique de l'euphémisme à valeur ironique qui permet à l'énonciateur de déterminer « de quel côté penchent les Français » dans le débat en question. La réponse nous est donnée implicitement : la sécurité, plutôt que la liberté. L'adjectif sempiternel, qui disqualifie la légitimité même du débat sur cette question, traduit bien la position adoptée. Le jeu des postures énonciatives est donc assez complexe : les formes de sous et co-énonciation sont contredites par les évaluatifs, il s'agit de faire passer le point de vue de l'éditorialiste comme celui qui exprime celui de l'ensemble des Français.

\section{Conclusion}

51 Le croisement des deux démarches, celle qui part des dénominations lexicales comme premier élément de construction d'un point de vue et celle qui s'intéresse aux postures qu'adoptent les énonciateurs nous a paru fructueuse. La première approche a permis de montrer comment les sous-catégorisations d'islam traduisent les débats en cours dans la société française, dont le discours de presse se fait à la fois l'écho et le producteur. Les dénominations « externes » sont de ce point de vue particulièrement intéressantes : elles ont des connotations négatives, qui semblent majoritaires en nombre et en fréquence (islam radical, islam intégriste, islam fondamentaliste...) ou, plus rarement des dénominations connotations positives (islam tolérant, islam modéré). Certaines dénominations se veulent plus objectivantes, qu'il s'agisse des notions employées par des historiens de l'islam (islam des Lumières), par les politiques ou les organismes religieux (islam de France, islam républicain) ou les sociologues (islam identitaire). Quelques-unes portent une axiologie ambivalente selon les énonciateurs: la dénomination islam des caves traduit les conditions difficiles dans lesquelles les musulmans sont parfois obligés de pratiquer leur religion, mais elle peut aussi connoter le caractère souterrain, et donc inquiétant des pratiques religieuses vues par ceux qui leur sont étrangers.

52 L'analyse lexicale menée sur l'ensemble du corpus a permis de mettre en évidence, en nous fondant sur les co-occurrents les plus régulièrement associés à l'expression, quatre isotopies principales dans le discours de presse : l'islam de France est divers et divisé, il reste à construire, il comporte cependant des représentants et des institutions, qui sont soumises à l'injonction de se mieux se définir et de se développer.

Corela, HS-32 | 2020 
L'analyse des postures énonciatives, menée à partir des éditoriaux, confirme de son côté l'importance de l'injonction vis-à-vis de l'islam de France, appelé à se structurer et à prendre conscience de ses responsabilités. L'éditorialiste adopte une posture générale de sur-énonciation par rapport aux discours cités ou représentés (qu'il s'agisse des représentants de l'islam ou de la classe politique). Cette posture principale de surplomb, qui semble assez naturelle au genre de l'éditorial, prend cependant des formes assez variées dans les trois éditoriaux plus particulièrement étudiés: l'éditorialiste de Libération part d'un point de vue clairement exprimé (c'est aux musulmans de combattre l'islamisme) tout en se défaussant sur l'opinion des risques encourus si ce programme n'était pas réalisé ; celui du Monde adopte une posture plus en retrait, en manifestant son accord avec les autorités qui cherchent à construire institutionnellement l'islam de France, l'éditorialiste se réservant cependant le soin in fine d'expliquer ce qui arriverait si ce programme échouait. Enfin, l'éditorialiste du Figaro n'adresse pas d'injonction aux représentants de l'islam, mais aux pouvoirs publics, chargés d'édicter les règles qui permettront seules d'éviter les dérives islamistes, tout en s'appuyant sur ce qu'il présente comme le point de vue majoritaire des Français, la primauté de la sécurité sur la liberté. Dans l'éditorial au moins, il n'y a pas symétrie entre les différentes postures énonciatives; le discours de l'autre est toujours utilisé pour mieux mettre en évidence le point de vue "surplombant» du journal.

54 Enfin, du point de vue méthodologique, l'articulation entre l'approche lexicale et l'approche énonciative, gagnera à être prolongée par une étude plus approfondie et plus fine des structures prédicatives ${ }^{23}$. Il sera intéressant d'analyser comment les expressions comportant un nom pivot ( $\mathrm{N}$ de $\mathrm{N}$, et $\mathrm{N}+\mathrm{ADJ}$ ) fonctionnent en tant que prédication première, seconde, et comment s'effectuent ainsi les jeux de dénomination et de redénomination.

\section{BIBLIOGRAPHIE}

Belkaïd, A. (2016). Réflexion sur « l'islam des Lumières », Le Monde diplomatique, Manières de voir 145, février mars 2016.

Belkaïd, A., Schmid, L. (2015). « Islam de France, islam en France », Esprit 2015/2 (Février), 49-53.

Fløttum, K. (1998). « The editorial: A heterogeneous genre ». In L. Lundquist, \& J. Quistgaard (dir.), LSP. Identity and Interface Research, Knowledge and Society vol. I. Copenhague, Business School, 221-230.

Fløttum, K. (2001). « Le discours rapporté dans l'éditorial ». Travaux de linguistique, 41, 107-115.

Kleiber, G. (1984). Dénomination et relations dénominatives, Langages, 76, 77-9.

Kleiber, G. (2001). Remarques sur la dénomination, Cahiers de praxématique, 36, [En ligne] document 1, mis en ligne le 01 janvier 2009, consulté le 02 octobre 2016. URL : http:// praxematique.revues.org/292 
Monte, M. (2009). Si marqueur d'altérité énonciative dans les si P extraprédicatives non conditionnelles, Langue française, 163, 99-119.

Rabatel, A. (2003). Le point de vue, entre langue et discours, description et interprétation : état de l'art et perspectives, Cahiers de praxématique, 43 7-23.

Rabatel, A. (2004). L'effacement énonciatif dans les discours rapportés et ses effets pragmatiques, Langages, 156, 3-17.

Rabatel, A. (2007). Les enjeux des postures énonciatives et de leur utilisation en didactique, Éducation et didactique, 1/2, 89-116.

Rabatel, A. (2012). Positions, positionnements et postures de l'énonciateur, TRANEL, Travaux Neuchâtelois de Linguistique, Institut des sciences du langage et de la communication (Neuchâtel, Suisse), 2012, 56, 23-42. <halshs-00769273>

Rabatel A. (2015a). Retour sur un parcours en énonciation. In M. Carcassonne, A. Cunha Doris, C. Donahue, F. François, A. Rabatel (dir.), Points de vue sur le point de vue. Un essai de réflexion collective, Limoges, Lambert-Lucas, 327-355.

Rabatel, A. (2015b). Postures énonciatives, variable générique et stratégies de positionnement. In J. Angermuller, G. Philippe (dir.), Analyse du discours et dispositifs d'énonciation. Autour des travaux de Dominique Maingueneau, Limoges, Lambert-Lucas, 125-136.

Ringoot, R. (2015). L'auctorialité journalistique. In J. Angermüller et G. Philippe (dir.), Analyse du discours et dispositifs d'énonciation. Autour des travaux de Dominique Maingueneau. Limoges, Lambert-Lucas.

Roitman, M. (2006). Polyphonie argumentative. Étude de la négation dans des éditoriaux du Figaro, de Libération et du Monde. Thèse, Institutionen för franska, italienska och klassiska språk, Département de français, d'italien et de langues classiques. Université de Stockholm.

Seniguer, H. (2012). Les catégories dénominatives de l'islam à l'épreuve d'un objet "mutant", Cahiers d'études africaines [En ligne], 206-207, mis en ligne le 01 juillet 2014, consulté le 18 février 2018. URL : http://journals.openedition.org/etudesafricaines/17106

Steuckardt, A. (2002). Les ennemis selon L'Ami du peuple, ou la catégorisation identitaire par contraste, Mots, Les langages du politique [En ligne], 69, mis en ligne le 13 mai 2008, consulté le 18 février 2018. URL : http://journals.openedition.org/mots/10023

\section{NOTES}

1. L'étude de corpus se fonde sur le travail de Master 2 d'Alice Pitoizet.

2. Nous donnons à la notion de point de vue son sens ordinaire : le terme traduit l'orientation argumentative d'un énoncé, telle qu'elle peut être repérée à travers un certain nombre de procédés, rhétoriques et linguistiques.

3. Nous remercions Moussa Bourekba, chercheur au Centre de Barcelone pour les Affaires Internationales et professeur associé en relations internationales à la Faculté de Blanquerna (Université Ramon Llul, Barcelone) ainsi que les autres membres de l'équipe du projet «L'islam, objet médiatique » d'avoir donné accès à Alice Pitoizet au corpus de presse constitué dans ce cadre.

4. Anatext est un outil d'analyse de corpus lexicométrique développé par Olivier Kraif à l'Université Grenoble Alpes : http://phraseotext.univ-grenoble-alpes.fr/anaText/ 
5. Belkaïd (2016) parle de l'islam des Lumières comme d'un «concept séduisant en apparence, mais dont le contenu reste à définir au-delà de la simple formule médiatique destinée à se démarquer de l'intégrisme. »

6. P. Karina, «L'impossible réforme de l'islam de France ", Courrier international, N 1440, 7 juin 2018.

7. Suleiman, Nasser, «Islam de France? Parlons plutôt d'islam français », Huffington Post - les blogs, 12 mars 2015.

8. Voir la transcription du débat sur http://presidentielle2012.ouest-france.fr/actualite/leverbatim-du-duel-entre-hollande-et-sarkozy-03-05-2012-1500

9. Auffray, Alain, « Non, Nicolas Sarkozy n'a pas “inventé" l'islam de France », Libération, 8 avril 2016, https://www.liberation.fr/france/2016/04/08/non-nicolas-sarkozy-n-a-pas-invente-lislam-de-france_144489

10. Sénat, Rapport $n^{\circ} 757$ : Rapport d'information fait au nom de la mission d'information sur l'organisation, la place et le financement de l'Islam en France et de ses lieux de culte, présenté par Nathalie Goulet, session extraordinaire, 5 juillet 2016.

11. A noter que la catégorie « journaliste » est assez hétérogène, puisqu'elle peut recouvrir, entre autres, des éditorialistes, des interviewers, ou encore des journalistes «de terrain» ayant effectué un reportage.

12. Rappelons que ce sous-corpus comprend 49 éditoriaux extraits du corpus principal, clairement identifiés en tant que tels, avec 68 mentions d'islam (pour environ 29460 mots).

13. L'étude en contexte nuance un peu ce constat: certaines dénominations (branche majoritaire vs minoritaire de l'islam) et le cadrage de certaines occurrences d'islam permettent dans un ou deux cas de spécifier une branche de l'islam : "La plupart des musulmans ne sont pas salafistes. Mais les salafistes sont une branche extrême, fondamentaliste, de l'Islam » (Libération, éditorial du 23/11/2015). On trouve parfois des formes plus vagues telle que "Pour s'être élevé, respectueusement, intelligemment, courageusement, contre cette version de l'islam, un jeune blogueur saoudien, Raef Badaoui, vient de voir sa peine confirmée en appel : dix ans de prison, dix ans d'interdiction de sortie du territoire et mille coups de fouet à distribuer en vingt semaines » (Le Monde, éditorial du 9/06/2015).

14. Sur la dénomination islam des Lumières, on consultera entre autres l'article d'Akram Belkaïd (2016).

15. Le ministère de l'Intérieur dénombrait, en 2010, environ 15.000 salafistes en France.

16. Ce syntagme ne correspond pas à une dénomination, et s'apparente plutôt à une désignation caractérisante, même si elle est utilisée par contraste à islam radical et islam salafiste qui fonctionnent comme des dénominations.

17. Voir par exemple dans cet éditorial du Figaro : «Aussi est-il impérieux de remettre l'histoire à l'endroit. Et de dénoncer ces nouveaux programmes scolaires qui estompent l'héritage de Louis XIV ou des Lumières pour lui préférer celui de l'Islam ou de l'empire du Mali. » (Le Figaro, 10/05/2015).

18. «Voilà pourquoi on réservera ici la notion de coénonciation à un échange dans lequel la colocution révèle un accord des deux locuteurs avec ce qu'ils énoncent, faisant de cet énoncé, coconstruit et pris en charge par les deux énonciateurs, l'expression d'un point de vue commun ».

19. Mais il ne nous est pas dit «ce qui lui semble inéluctable», ce qui encourage le lecteur à conclure à une prise en charge de l'assertion par L1/E1.

20. Même si elle ne termine pas l'éditorial, qui comporte encore les phrases suivantes: «Hier soir, invité du journal de France 2 par David Pujadas, dont il a fait un personnage de son roman, Houellebecq a rajouté au trouble. Il a gommé la frontière entre la fiction et la réalité. Le constat est violent ; mais la voie douce. »

21. On peut penser d'ailleurs qu'il y a surinterprétation et gauchissement de la portée du roman, l'interprétation du roman de Houellebecq étant sans doute plus ouverte. 
22. Elle apparaît quatre fois (dont deux dans l'éditorial de Libération), contre deux pour islam français, et une pour islam en France.

23. Nous remercions Alain Rabatel d'avoir attiré notre attention sur ce point, ainsi que de sa relecture constructive de notre article.

\section{RÉSUMÉS}

Durant la période récente, marquée par les attentats de terroristes islamistes, la presse française s'est trouvée face à des problèmes de dénomination délicats, liés à l'utilisation du terme islam et à ses sous-catégorisations (islam radical, islam modéré, islam religieux, islam politique ; islam de France...) qui entrent dans un micro-système terminologique en voie de constitution. Cependant, ces dénominations ne sont pas toujours stabilisées dans un sens précis, et leur contexte d'énonciation, tout comme le point de vue des énonciateurs, est déterminant pour leur interprétation. A travers l'étude d'un corpus regroupant les articles des journaux Le Monde, Le Figaro et Libération entre le 1er janvier 2014 et le 31 décembre 2015, contenant de nombreuses occurrences des mots islam et musulman, la recherche présentée analyse les postures énonciatives qui apparaissent dans la construction et l'emploi des catégorisations/désignations de l'islam dans la presse française, et plus particulièrement chez les éditorialistes.

In a context marked by the terrorist islamist attacks, French press had recently experienced difficulties in the sub-classification of Islam (e.g. radical Islam, moderate Islam, religious Islam, political Islam, Islam of France...). However, even though such terms are very common, they are seldom used with any precision or a clear grasp of their meaning. Therefore, the enunciation context, as well as the enunciators' point of view, are both significant determinants for their interpretation. Through the study of a corpus gathered from three well known French newspapers (Le Monde, Le Figaro, Libération), between January 1, 2014 and December 31, 2015, including many occurrences of the words islam and musulman (muslim), our paper analyzes the relations between lexical designations and enunciative postures, implied by the subcategorizations of islam in the French press, and more particularly among editorial writers.

\section{INDEX}

Mots-clés : terminologie, dénomination, posture énonciative, islam, discours de presse, éditorial Keywords : terminology, lexical designation, enunciative posture, islam, press discourse, editorial

\section{AUTEURS}

\section{ALICE PITOIZET}

Lidilem, Univ. Grenoble Alpes 